\title{
On Multiple Node Gaussian Quadrature Formulae*
}

\author{
By David L. Barrow
}

Abstract. Let $\mu_{1}, \ldots, \mu_{k}$ be odd positive integers and $n=\Sigma_{i=1}^{k}\left(\mu_{i}+1\right)$. Let $\left\{u_{i}\right\}_{i=1}^{n}$ be an extended Tchebycheff system on $[a, b]$. Let $L$ be a positive linear functional on $U \equiv \operatorname{span}\left(\left\{u_{i}\right\}\right)$. We prove that $L$ has a unique representation in the form

$$
L(p)=\sum_{i=1}^{k} \sum_{j=0}^{\mu_{i}-1} a_{i j} p^{(j)}\left(t_{i}\right), \quad a<t_{1}<\cdots<t_{k}<b,
$$

for all $p \in U$. The proof uses the topological degree of a mapping $F: \bar{D} \subset R^{k}$ $\rightarrow R^{k}$. The result is proved by showing that the equation $F(\underline{t})=0$ has a unique solution, which in turn is proved by showing that $F$ has degree 1 and that for any solution $\underline{t}$ to the equation $F(\underline{t})=0$, det $F^{\prime}(\underline{t})>0$. We also give extensions to the cases when the $\left\{u_{i}\right\}$ are a periodic extended Tchebycheff system and when $L$ is a nonnegative linear functional.

1. Introduction. A quadrature formula is an approximation to a definite integral by a linear combination of values of the integrand and its derivatives at selected points. It is a classical result that the formula

$$
\sum_{i=1}^{k} a_{i} f\left(t_{i}\right) \simeq \int_{a}^{b} f(t) \phi(t) d t, \quad \phi(t) \geqslant 0,
$$

can, by a judicious choice of the points $a<t_{1}<\cdots<t_{k}<b$ and weights $\left\{a_{i}\right\}$, be made exact for all integrands $f$ which are polynomials of degree at most $2 k-1$. Furthermore, this choice of points and weights is unique. An equivalent statement is that a certain system of $2 k$ equations in the $2 k$ unknowns $\left\{t_{i}\right\},\left\{a_{i}\right\}$ has a unique solution. Formulas of this type, and their various generalizations, are usually known as Gaussian quadrature formulas.

In [1], Karlin and Pinkus give a discussion of some of these generalizations. We refer the reader to that paper and its excellent bibliography for more information on this subject.

This paper is concerned with generalizations of the following theorem, which is proved in [1].

THEOREM A. Let $\mu_{1}, \ldots, \mu_{k}$ be odd positive integers, and let $n=\Sigma_{i=1}^{k}\left(\mu_{i}+1\right)$. Let $\left\{u_{i}\right\}_{i=1}^{n}$ be an extended complete Tchebycheff system on the interval $[a, b]$. Then there are unique points $a<t_{1}<\cdots<t_{k}<b$ and coefficients $\left\{a_{i j}\right\}_{i=1}^{k}{\underset{j}{i}=0}_{0}^{-1}$ such that

Received November 15, 1976.

AMS (MOS) subject classifications (1970). Primary 65D30; Secondary 41 A55.

* This work was partially supported by NSF Grant DC R75-04545. 


$$
\sum_{i=1}^{k} \sum_{j=0}^{\mu_{i}-1} a_{i j} u_{m}^{(j)}\left(t_{i}\right)=\int_{a}^{b} u_{m}(t) \phi(t) d t, \quad \phi(t) \geqslant 0,
$$

holds for $m=1,2, \ldots, n$.

This theorem was proved by extending the $\left\{u_{i}\right\}$ to a larger interval $(c, d) \supset$ $[a, b]$ and then using induction on the number of points $\left\{t_{i}\right\}$ inside $(a, b)$. The implicit function theorem and the completeness of the system $\left\{u_{i}\right\}$ (see Section 2 for definitions) were crucial ingredients in the proof.

The present paper gives an entirely different proof of this theorem, using the topological degree of a mapping $F: D \subset R^{k} \rightarrow R^{k}$. This proof is somewhat simpler, and obviates the requirement that the system $\left\{u_{i}\right\}$ be complete. This in turn makes an extension of the theorem to periodic Tchebycheff systems straightforward, an extension whose proof is apparently not possible by the method used in [1]

In Section 2 we present some definitions and some results on Tchebycheff systems and degree theory which will be needed in our subsequent analysis. The main result, Theorem 1, is proved in Section 3. The generalizations of this theorem to the periodic case (Theorem 2) and to nonnegative linear functionals (Theorem 3) are presented in Section 4 , along with a brief discussion on reducing the smoothness required of the $\left\{u_{i}\right\}$.

2. Definitions and Preliminary Results. Let $\left\{u_{i}\right\}_{i=1}^{n}$ be functions of class $C^{N-1}[a, b]$. Then the $\left\{u_{i}\right\}$ are said to be an extended Tchebycheff system (ET-system) of order $N$ (see [2, p. 6]) provided

$$
\operatorname{det}\left[\begin{array}{cc}
u_{1}\left(t_{1}\right) & u_{1}\left(t_{2}\right) \cdots u_{1}\left(t_{n}\right) \\
u_{2}\left(t_{1}\right) & u_{2}\left(t_{2}\right) \cdots u_{2}\left(t_{n}\right) \\
\vdots & \\
\cdot & \\
u_{n}\left(t_{1}\right) & u_{n}\left(t_{2}\right) \cdots u_{n}\left(t_{n}\right)
\end{array}\right]^{*}>0
$$

whenever $a \leqslant t_{1} \leqslant t_{2} \leqslant \cdots \leqslant t_{n} \leqslant b$. The "** means that if some of the $t_{i}$ 's coincide, then the columns in the matrix corresponding to coincident $t_{i}$ 's are replaced by derivatives, of increasing order. Thus, if $n=3$ and $t_{1}=t_{2}<t_{3}$, the condition is

$$
\operatorname{det}\left[\begin{array}{lll}
u_{1}\left(t_{1}\right) & u_{1}^{\prime}\left(t_{1}\right) & u_{1}\left(t_{3}\right) \\
u_{2}\left(t_{1}\right) & u_{2}^{\prime}\left(t_{1}\right) & u_{2}\left(t_{3}\right) \\
u_{3}\left(t_{1}\right) & u_{3}^{\prime}\left(t_{1}\right) & u_{3}\left(t_{3}\right)
\end{array}\right]>0
$$

At most $N$ consecutive $t_{i}$ 's are allowed to coincide. An easy consequence of this definition is the following fact, which we will use extensively: if the points $a \leqslant t_{1}$ $<\cdots<t_{k} \leqslant b$, the nonnegative integers $\left\{\mu_{i}\right\}_{i=1}^{k}, \mu_{i} \leqslant N-1$, and the data $\left\{c_{i j}\right\}_{i=1}^{k} \mu_{j=0}^{\mu_{i}}$ are prescribed, and if $n=\Sigma_{i=1}^{k}\left(\mu_{i}+1\right)$, then there is a unique "polynomial" $p(t)=\sum_{i=1}^{n} \alpha_{i} u_{i}(t)$ which satisfies $p^{(j)}\left(t_{i}\right)=c_{i j}, i=1, \ldots, k, j=0,1, \ldots$, 
$\mu_{i}$. Hence, any nontrivial polynomial $p(t)$ can have at most $n-1$ zeros, counting multiplicities up to order $N$.

An ET-system of order $n$ is called simply an extended Tchebycheff (ET) system. If the functions $\left\{u_{i}\right\}_{i=1}^{n}$ satisfy the property that for any $k, 1 \leqslant k \leqslant n$, the system $\left\{u_{i}\right\}_{i=1}^{k}$ is an ET-system, then we say the $\left\{u_{i}\right\}_{i=1}^{n}$ form an extended complete Tchebycheff $(E C T)$ system.

Let $\left\{u_{i}\right\}_{i=1}^{n}$ be an ET-system, and let $U=$ (real) $\operatorname{span}\left(\left\{u_{i}\right\}\right)$. A linear functional $L$ on $U$ is said to be positive if whenever $p \in U, p \geqslant 0, p \neq 0$, then $L(p)>0$. $L$ is called nonnegative if $L(p) \geqslant 0$ whenever $p \geqslant 0$. It is shown in [3] (Theorem 1.1) that a nonnegative linear functional $L$ on $U$ may be extended to a nonnegative linear functional $\widetilde{L}$ on $C[a, b]$. Hence, using the Riesz representation theorem for the dual of $C[a, b]$, we may assume that $L$ has the form $L(p)=\int_{a}^{b} p(t) d \mu(t), p \in U$, where $\mu(t)$ is a nondecreasing right continuous bounded function. At this point we prove a proposition, a form of which is stated in [1], which shows that Theorem 1 (below) is sharp.

Proposition 1. Let $\left\{u_{i}\right\}_{i=1}^{n}$ be an ET-system on $(c, d) \supset[a, b]$, and let $L$ be a positive linear functional on $\left.U\right|_{[a, b]}$, the restriction of functions in $U=\operatorname{span}\left(\left\{u_{i}\right\}\right)$ to $[a, b]$. If $c<t_{1}<\cdots<t_{k}<d$ and if $\left\{\mu_{i}\right\}_{i=1}^{k}$ are positive integers, then if

$$
\sum_{i=1}^{k} \sum_{j=0}^{\mu_{i}-1} a_{i j} u_{m}^{(j)}\left(t_{i}\right)=L\left(u_{m}\right)
$$

for $m=1,2, \ldots, n$, it must be that $n \leqslant \vec{n} \equiv \Sigma_{i=1}^{k} \mu_{i}+F$, where $F$ is the number of points $t_{i}$ such that $t_{i} \in(a, b)$ and $\mu_{i}$ is odd.

Proof. Suppose that $n>\bar{n}$. Let $p \in U$ be nontrivial and satisfy:

$$
\begin{array}{ll}
p^{(j)}\left(t_{i}\right)=0, j=0, \ldots, \mu_{i} & \text { if } t_{i} \in(a, b) \text { and } \mu_{i} \text { is odd; } \\
p^{(j)}\left(t_{i}\right)=0, j=0, \ldots, \mu_{i}-1 \quad \text { for all other } t_{i} \text { 's. }
\end{array}
$$

This is a total of $\bar{n}$ zeros. Let $p$ have an additional $n-\bar{n}-1$ zeros outside $(a, b)$ if $n>\bar{n}+1$; then $p$ can have no other zeros. Note that all zeros of $p$ which are interior to $(a, b)$ have even multiplicity; hence $p$ does not change sign on $[a, b]$, so that $L(p)$ $\neq 0$. This is seen to be a contradiction of (3).

We next discuss the elements of topological degree theory which will be needed (cf. Schwartz [4, Chapter 3]). Let $D \subset R^{n}$ be a bounded open set, and let $F: \bar{D} \rightarrow$ $R^{n}$ be continuous. Then if $c \in R^{n}$ and $c \notin F(\partial D)(\partial D=$ boundary of $D)$, the degree of $F$ with respect to $D$ and $c$ is defined, is an integer, and will be denoted $\operatorname{deg}(F, D, c)$. The following are some properties of the degree:

(i) If $F \in C^{1}(D) \cap C(\bar{D}), c \notin F(\partial D)$, and $\operatorname{det}\left(F^{\prime}(x)\right) \neq 0$ when $F(x)=c$, there are a finite number of points $x_{i} \in D$ where $F\left(x_{i}\right)=c$, and $\operatorname{deg}(F, D, c)=$ $\Sigma_{i} \operatorname{sgn}\left(\operatorname{det}\left(F^{\prime}\left(x_{i}\right)\right)\right)$.

(ii) If $\operatorname{deg}(F, D, c) \neq 0$, there is at least one solution in $D$ to the equation $F(x)$ $=c$.

(iii) If $F: \bar{D} \times[0,1] \rightarrow R^{n}$ is continuous and $F(x, \lambda) \neq c$ for $x \in \partial D, 0 \leqslant \lambda$ $\leqslant 1$, then $\operatorname{deg}(F(\cdot, \lambda), D, c)$ is constant, independent of $\lambda$. 
Let $\Delta_{k}$ be the open simplex

$$
\Delta_{k}=\left\{\underline{t}=\left(t_{1}, \ldots, t_{k}\right): a<t_{1}<\cdots<t_{k}<b\right\},
$$

and let

$$
\begin{aligned}
\Delta_{k, \epsilon}=\left\{\underline{t}=\left(t_{1}, \ldots, t_{k}\right): a=t_{0}<t_{1}<\cdots<t_{k}<t_{k+1}=b,\right. \\
\\
\left.\quad t_{i+1}-t_{i}>\epsilon, i=0,1, \ldots, k\right\} .
\end{aligned}
$$

Finally, it will be convenient to let $\underline{a}=\left(a_{10}, a_{11}, \ldots, a_{1 \mu_{1-1}}, a_{20}, a_{21}, \ldots\right.$, $\left.a_{2 \mu_{2}-1}, \ldots, a_{k \mu_{k}-1}\right)$, where $\mu_{1}, \mu_{2}, \ldots, \mu_{k}$ are prescribed positive integers.

3. Statement and Proof of Theorem 1. Let $\mu_{1}, \ldots, \mu_{k}$ be odd positive integers, and let $n=\Sigma_{i=1}^{k}\left(\mu_{i}+1\right)$. Let $\left\{u_{i}\right\}_{i=1}^{n}$ be an ET-system on $[a, b]$, and let $L$ be a positive linear functional on $U \equiv \operatorname{span}\left(\left\{u_{i}\right\}\right)$.

THEOREM 1. There is a unique $\underline{t} \in \Delta_{k}$ and coefficient vector $\underline{a}$ such that

$$
\sum_{i=1}^{k} \sum_{j=0}^{\mu_{i}-1} a_{i j} p^{(j)}\left(t_{i}\right)=L(p)
$$

for all $p \in U$.

The proof will be based on a series of lemmas.

Lemma 1. Let $\underline{t} \in \Delta_{k}$, and for $i=1, \ldots, k, j=0, \ldots, \mu_{i}$, let $p_{i j}(\underline{t} ; \cdot) \in U$ satisfy $p_{i j}^{(j)}\left(\underline{t} ; t_{i}\right)=1$ and $p_{i j}^{(m)}\left(\underline{t} ; t_{l}\right)=0$ if $(l, m) \neq(i, j), m=0, \ldots, \mu_{l}, l=1, \ldots$, k. Let $a_{i j}=L\left(p_{i j}(\underline{t} ; \cdot)\right), i=1, \ldots, k, j=0, \ldots, \mu_{i}-1$. Then $\underline{t} \underline{a}$ satisfy (4) if and only if

$$
L\left(p_{i \mu_{i}}(t ; \cdot)\right)=0, \quad i=1, \ldots, k .
$$

Proof. If (4) holds, (5) is trivial. Conversely, if (5) holds, then (4) holds for all $p_{i j}(t ; \cdot)$, and hence for all $p \in U$, since the $p_{i j}(t ; \cdot)$ form a basis for $U$.

Lemma 2. If $\underline{t}, \underline{a}$ satisfy (4), then $a_{i \mu_{i-1}}>0, i=1, \ldots, k$.

Proof. For $i=1, \ldots, k$, let $p_{i} \in U$ satisfy

$$
\begin{aligned}
& p_{i}^{(j)}\left(t_{l}\right)=0, \quad j=0, \ldots, \mu_{l}, l=1, \ldots, k, l \neq i ; \\
& p_{i}^{\left(\mu_{i}-1\right)}\left(t_{i}\right)=1 ; \\
& p_{i}^{(j)}\left(t_{i}\right)=0, \quad j=0, \ldots, \mu_{i}-2 \text { if } \mu_{i}>1 ; \\
& p_{i}(a)=0 .
\end{aligned}
$$

Then $p_{i}$ has $n-1$ zeros and hence no others. All zeros of $p_{i}$ which are interior to $(a, b)$ have even multiplicity, and so $p_{i} \geqslant 0$. Hence

$$
a_{i \mu_{i}-1}=L\left(p_{i}\right)>0 \text {. }
$$

Now fix $\underline{r}=\left(r_{1}, \ldots, r_{k}\right) \in \Delta_{k}$, and define $L_{0} \in U^{*}$ (the dual of $U$ ) by

$$
L_{0}(p)=\sum_{i=1}^{k} p\left(r_{i}\right)
$$

Let $L_{\lambda}=(1-\lambda) L_{0}+\lambda L, 0 \leqslant \lambda \leqslant 1$.

Lemma 3. There is an $\epsilon>0$ such that whenever $\underline{t}_{\lambda}, \underline{a}_{\lambda}$ satisfy (4) for $L_{\lambda}$, then $\underline{t}_{\lambda} \in \Delta_{k, \epsilon}$. 
Proof. Suppose no such $\epsilon$ exists. Then there is a sequence $\left\{\lambda_{m}, \underline{t} \lambda_{m} \equiv \underline{t}^{m}\right\}$ such that as $m \rightarrow \infty, \lambda_{m} \rightarrow \lambda_{0}$ and $\underline{t}^{m} \rightarrow \underline{t} \in \partial \Delta_{k}$. For ease of exposition, we assume that $a<t_{1}=t_{2}<t_{3}<\cdots<t_{k}<b$. The other possibilities for $\underline{t} \in \partial \Delta_{k}$ can be handled similarly. We will construct a sequence of polynomials $\left\{p_{m}\right\}$ such that $L_{\lambda_{m}}\left(p_{m}\right)=0, p_{m} \rightarrow p$ uniformly as $m \rightarrow \infty$, but $L_{\lambda_{0}}(p)>0$, a contradiction.

Let $p_{m} \in U$ satisfy

$$
\begin{aligned}
& p_{m}^{(j)}\left(t_{i}^{m}\right)=0, \quad j=0, \ldots, \mu_{i}, i=3, \ldots, k \\
& p_{m}^{(j)}\left(t_{i}^{m}\right)=0, \quad j=0, \ldots, \mu_{i}-1, i=1,2 ; \\
& p_{m}(a)=0, \quad p_{m}^{\prime}(a)>0, \quad\left\|p_{m}\right\| \equiv \max \left|p_{m}(t)\right|=1 .
\end{aligned}
$$

Clearly, $L_{\lambda_{m}}\left(p_{m}\right)=0$, since $\underline{t}^{m}, \underline{a}_{\lambda_{m}}$ give a formula (4) for $L_{\lambda_{m}}$.

Now let $p \in U$ satisfy

$$
\begin{aligned}
& p^{(j)}\left(t_{i}\right)=0, \quad j=0, \ldots, \mu_{i}, i=3, \ldots, k \\
& p^{(j)}\left(t_{1}\right)=0, \quad j=0, \ldots, \mu_{1}+\mu_{2}-1 ; \\
& p(a)=0, \quad p^{\prime}(a)>0, \quad\|p\|=1 .
\end{aligned}
$$

Now since the $\left\{p_{m}\right\}$ are uniformly bounded and belong to a finite dimensional vector space, a subsequence, call it $\left\{p_{m}\right\}$ again, must converge, $p_{m} \rightarrow q$, the convergence being in the topology of $C^{n-1}[a, b]$. We claim that $p$ and $q$ have the same zeros, and hence that $p=q$, by the normalizations on $p$ and the $p_{m}$.

Repeated application of Rolle's Theorem to $p_{m}$ shows there are points $\left\{\xi_{j}^{m}\right\} \subset$ $\left[t_{1}^{m}, t_{2}^{m}\right]$ such that $p_{m}^{(j)}\left(\xi_{j}^{m}\right)=0, j=0, \ldots, \mu_{1}+\mu_{2}-1$. It follows that $q^{(j)}\left(t_{1}\right)=$ $\lim _{m \rightarrow \infty} p_{m}^{(j)}\left(t_{1}\right)=0, j=0, \ldots, \mu_{1}+\mu_{2}-1$. It is clear then that $p$ and $q$ have the same zeros and so $p=q$. It remains to show that $L_{\lambda_{0}}(p)>0$. Arguing as before, we see that $p \geqslant 0$ on $[a, b]$, and hence $L_{\lambda_{0}}(p)>0$ if $\lambda_{0} \neq 0$. If $\lambda_{0}=0$, it again follows that $L_{\lambda_{0}}(p)>0$, since $\underline{t} \neq \underline{r}$. This completes the proof of Lemma 3 .

Let $\epsilon>0$ be as in Lemma 3. For $\underline{t} \in \Delta_{k, \epsilon}$, let $p_{i}(\underline{t} ; \cdot) \in U$ be the polynomial $p_{i \mu_{i}}(\underline{t} ; \cdot)$ of Lemma 1 . We define the map $F: \Delta_{k, \epsilon} \times[0,1] \rightarrow R^{k}$ by

$$
F_{i}(t, \lambda)=-L_{\lambda}\left(p_{i}(t ; \cdot)\right), \quad i=1, \ldots, k .
$$

Then $F$ is continuous in $(\underline{t}, \lambda)$ and Lemmas 1 and 3 imply that $F(\underline{t}, \lambda) \neq 0$ if $\underline{t} \in$ $\partial \Delta_{k, \epsilon}$. Hence $\operatorname{deg}\left(F(\cdot, \lambda), \Delta_{k, \epsilon}, 0\right)$ is defined and is independent of $\lambda$, by property (iii) of the degree. We will show that this degree is one, and conclude that there is a unique solution to the equation $F(\underline{t}, 1)=0$. This will complete the proof of Theorem 1.

LEMMA 4. If $\underline{t} \in \Delta_{k, \epsilon}$ is any solution to the equation $F(\underline{t}, \lambda)=0,0<\lambda \leqslant 1$, then

$$
\operatorname{det} \frac{\partial F}{\partial \underline{t}}(\underline{t}, \lambda)>0 \text {. }
$$

Proof. For convenience we delete the explicit reference to the parameter $\lambda$.

Since $F(\underline{t})=0$, let $\underline{t} \underline{a}$ be the formula (4) guaranteed by Lemma 1 . Let $|h|$ be small, and let $\underline{e}_{1}=(1,0, \ldots, 0) \in R^{k}$. Then 


$$
\begin{aligned}
F_{1}\left(\underline{t}+h \underline{e}_{1}\right)-F_{1}(\underline{t})=-L\left(p_{1}\left(\underline{t}+h \underline{e}_{1} ; \cdot\right)\right) \\
=-\sum_{i=1}^{k} \sum_{j=0}^{\mu_{i}-1} a_{i j} p_{1}^{(j)}\left(\underline{t}+h \underline{e}_{1} ; t_{i}\right) \\
=-\sum_{j=0}^{\mu} a_{1 j} p_{1}^{(j)}\left(\underline{t}+h \underline{e}_{1} ; t_{1}\right)=-\sum_{j=0}^{\mu_{1}^{-1}} a_{1 j}\left(\frac{(-h)^{\mu}-j}{\left(\mu_{1}-j\right) !}+o\left(h^{\mu_{1}-j}\right)\right),
\end{aligned}
$$

the last equality following from a Taylor expansion of $p_{1}^{(j)}\left(\underline{t}+h \underline{e}_{1} ; t_{1}\right)$ about $t_{1}+h$. Therefore,

$$
\frac{\partial F_{1}}{\partial t_{1}}(\underline{t})=\lim _{h \rightarrow 0} \frac{F_{1}\left(\underline{t}+h \underline{e}_{1}\right)-F_{1}(\underline{t})}{h}=a_{1 \mu_{1}-1} .
$$

By similar reasoning, one shows that

$$
\frac{\partial F}{\partial \underline{t}}(\underline{t})=\operatorname{diag}\left(a_{1 \mu_{1}-1}, \ldots, a_{k \mu_{k^{-1}}}\right),
$$

which, in view of Lemma 2, proves the lemma.

Lemma 5. $\operatorname{Deg}\left(F(\cdot, 0), \Delta_{k, \epsilon}, 0\right)=1$.

Proof. We again delete explicit reference to the parameter $\lambda=0$, so that

$$
F_{i}(t)=-L_{0}\left(p_{i}(\underline{t} ; \cdot)\right)=-\sum_{l=1}^{k} p_{i}\left(\underline{t} ; r_{l}\right)
$$

It is clear that $F(\underline{r})=0$, and Lemma 3 implies that $\underline{r} \in \Delta_{k, \epsilon}$. We claim that $\underline{r}$ is the only solution to $F(\underline{t})=0$. For suppose that $\underline{s} \in \Delta_{k, \epsilon}$ also satisfies $F(\underline{s})=0$. Then by Lemma 1 (which holds for $L_{0}$ also) let $\underline{s}, \underline{a}$ be the formula (4) for $L_{0}$. Suppose $r_{1} \neq s_{1}$. Construct $p \in U$ to satisfy

$$
\begin{aligned}
& p^{(j)}\left(s_{i}\right)=0, \quad j=0, \ldots, \mu_{i}-1, i=1, \ldots, k \\
& p\left(r_{i}\right)=0, \quad i=2, \ldots, k \\
& p\left(r_{1}\right) \neq 0 .
\end{aligned}
$$

Then (4) implies $L_{0}(p)=0$, but $L_{0}(p)=p\left(r_{1}\right) \neq 0$, a contradiction. Hence, $s_{1}=r_{1}$, and one similarly shows $\underline{s}=\underline{r}$.

Now, unfortunately, det $\partial F(\underline{r}) / \partial \underline{t}=0$ (if some $\mu_{i}>1$, as can be seen from (8), below), so a little care must be used in calculating the degree. We claim that $F(\underline{t})$ is $1-1$ for $\underline{t}$ near $\underline{r}$. For $\underline{t}$ near $\underline{r}$, we have

$$
F_{i}(\underline{t})=-\sum_{l=1}^{k} p_{i}\left(\underline{t} ; r_{l}\right)=-\frac{\left(r_{i}-t_{i}\right)^{\mu_{i}}}{\mu_{i} !}+\sum_{l=1}^{k} o\left(\left(r_{l}-t_{l}\right)^{\mu} l\right) .
$$

If we make the invertible, orientation preserving change of variables $\bar{t}_{i}=\left(t_{i}-r_{i}\right)^{\mu_{i}}$, we have 


$$
F_{i}(\bar{t})=\frac{\bar{t}_{i}}{\mu_{i} !}+\sum_{l=1}^{k} o\left(\bar{t}_{l}\right) .
$$

Hence, $\partial F(0) / \partial \underline{\underline{t}}=\operatorname{diag}\left(1 / \mu_{1} !, \ldots, 1 / \mu_{k} !\right)$, and so by the inverse function theorem, $F(\underline{\bar{t}})$ is $1-1$ for $\underline{\bar{t}}$ near 0 , and hence $F(\underline{t})$ is $1-1$ for $\underline{t}$ near $\underline{r}$. To complete the argument, we use the fact that $\operatorname{deg}\left(F, \Delta_{k, \epsilon}, 0\right)=\operatorname{deg}\left(F, \Delta_{k, \epsilon}, c\right)$ for $c$ near $0[4, \mathrm{p} .72]$ and the latter degree is easily seen to be one.

Theorem 1 now follows easily. Lemma 5 and property (iii) of the degree imply $\operatorname{deg}\left(F(\cdot, 1), \Delta_{k, \epsilon}, 0\right)=1$. Combining properties (i) and (ii) of the degree with Lemma 4 , we conclude that the equation $F(\underline{t}, 1)=0$ has a unique solution. Theorem 1 now follows from Lemma 1.

4. Extensions. A periodic extended Tchebycheff system is a set $\left\{u_{i}\right\}_{i=1}^{n} \subset$ $C^{n-1}(-\infty, \infty)$ of periodic functions having period $\tau$, such that their restriction to any interval $[a, a+\tau)$ form an ET-system on that interval. An example is the set $\{1$, $\cos t, \sin t, \ldots, \cos m t, \sin m t\}$. It is clear that such a set is not an ECT-system, since, for example the polynomial $1 / 2+\cos t$ has two zeros on $[0,2 \pi)$.

The appropriate generalization of Theorem 1 is the following:

THEOREM 2. Let $\mu_{1}, \ldots, \mu_{k}$ be odd positive integers and let $n=\sum_{i=1}^{k}\left(\mu_{i}+1\right)$ - 1. Let $\left\{u_{i}\right\}_{i=1}^{n}$ be a periodic ET-system with period $\tau$. Let $U=\operatorname{span}\left(\left\{\left.u_{i}\right|_{[0, \tau)}\right\}\right)$, and let $L$ be a positive linear functional on $U$. Let $t_{1} \in[0, \tau)$. Then there are unique points $\underline{t}=\left(t_{2}, \ldots, t_{k}\right), t_{1}<t_{2}<\cdots<t_{k}<t_{1}+\tau$ and coefficients $\underline{a}$ such that

$$
\sum_{i=1}^{k} \sum_{j=0}^{\mu_{i}-1} a_{i j} p^{(j)}\left(t_{i}\right)=L(p)
$$

for all $p \in U$.

Proof. The proof is the same as for Theorem 1, with minor modifications. Let $\Delta_{k-1}$ be the simplex

$$
\Delta_{k-1}=\left\{\underline{t}=\left(t_{2}, \ldots, t_{k}\right): t_{1}<t_{2}<\cdots<t_{k}<t_{1}+\tau\right\} .
$$

For $\underline{t} \in \Delta_{k-1}$ and $i=2, \ldots, k$, let $p_{i}(\underline{t} ; \cdot) \in U$ satisfy

$$
\begin{aligned}
& p_{i}^{(j)}\left(\underline{t} ; t_{l}\right)=0, \quad j=0, \ldots, \mu_{l}, l \neq 1, i \\
& p_{i}^{(j)}\left(\underline{t} ; t_{l}\right)=0, \quad j=0, \ldots, \mu_{l}-1, l=1, i, p_{i}^{\left(\mu_{i}\right)}\left(\underline{t} ; t_{i}\right)=1 .
\end{aligned}
$$

The theorem is proved by showing that there is a unique solution $\underline{t} \in \Delta_{k-1}$ to the equations $L\left(p_{i}(\underline{t} ; \cdot)\right)=0, i=2, \ldots, k$, and this is done just as before.

We sketch the changes in the Lemmas 1--5 which are required. The notation $i=1, \ldots, k$ is changed to $i=2, \ldots, k$ in obvious places (e.g. (5) and (6)). In Lemma 1 , the function $p_{1 \mu_{1}}(\underline{t})$ is deleted from the basis. In Lemmas 2 and 3 , the polynomials $p_{i}, p_{m}$, and $p$ should have a zero of (even) multiplicity $\mu_{1}+1$ at $t_{1}$, and the zero at $a$ is deleted. The functional $L_{0}$ is defined as before, with $r_{1}=t_{1}$ and $\underline{r}=$ $\left(r_{2}, \ldots, r_{k}\right) \in \Delta_{k-1}$. 
Another easy extension is to the case where $L$ is merely a nonnegative linear functional.

TheOREM 3. Let the hypotheses of Theorem 1 hold, except that $L$ is now any nonnegative linear functional. Then $L$ has a unique representation in the form.

$$
\sum_{i=1}^{k} \sum_{j=0}^{\mu_{i}-1} a_{i j} p^{(j)}\left(t_{i}\right)=L(p)
$$

for all $p \in U$, where $a \leqslant t_{1}<t_{2}<\cdots<t_{k} \leqslant b$.

Proof. We clearly may assume that $L$ is nontrivial and not positive. Thus, there is some nontrivial $q \in U, q \geqslant 0$, such that $L(q)=0$. Since

$$
L(p)=\int_{a}^{b} p(t) d \mu(t), \quad p \in U,
$$

for some nontrivial Stieltjes measure $d \mu(t), q$ must have zeros $a \leqslant s_{1}<s_{2}<\cdots<$ $s_{N} \leqslant b$. Furthermore, the support of $d \mu(t)$ must be contained in this set, so that

$$
L(p)=\sum_{i=1}^{N} \lambda_{i} p\left(s_{i}\right),
$$

where $\lambda_{i}>0$ (we discard and reindex if some $\lambda_{i}=0$ ). We consider two cases.

Case (i) $N \leqslant k$. In this case (12) is already in the form (11), and we have only to establish uniqueness. But this is easily done, by arguing as in Lemma 5.

Case (ii) $N>k$. This case is handled exactly as though $L$ were a positive linear functional. Indeed, the only use in the proof of Theorem 1 of the fact that $p \geqslant 0$ implies $L(p)>0$ was in Lemmas 2 and 3. In Lemma 3, the zero set of $p$ consisted of $k$ or fewer points, and so we have $L(p)>0$ in this case by (12). In Lemma 2 , we again have $L\left(p_{i}\right)>0$ by (12), unless $s_{1}=a, s_{2}=t_{1}, \ldots, s_{N}=t_{k}$. In this case we let $p_{i}$ satisfy all conditions of Lemma 2 , except that we replace $p_{i}(a)=0$ by $p_{i}(b)=0$. Then necessarily $L\left(p_{i}\right)=\lambda_{1} p_{i}(a)>0$. We remark that in this case $(N>k)$, the stronger conclusion of Theorem 1 holds, and all the $a_{i \mu_{i}-1}$ are positive.

We conclude with a conjecture, which is based on the following observation: if all $\mu_{i}=1$, then Theorems 1 and 2 are known to be true whenever $\left\{u_{i}\right\}_{i=1}^{n}$ is merely a Tchebycheff system (i.e., an ET-system of order one), with no smoothness requirements beyond continuity. This suggests the

Conjecture. Let the hypotheses of Theorem 1 hold, except that the $\left\{u_{i}\right\}_{i=1}^{n}$ are merely an extended Tchebycheff system of order $N$ where $N=\max \left(\mu_{i}\right)$. Then the conclusion of Theorem 1 holds. An analogous statement is true for Theorem 2.

We remark that the existence of the desired formula follows fairly easily from Theorem 1, since one may approximate the system $\left\{u_{i}\right\}$ by ET-systems, arbitrarily, closely (for example, as in [2, p. 15]). 
1. S. KARLIN \& A. PINKUS, "Gaussian quadrature formulae with multiple nodes," Studies in Spline Functions and Approximation Theory, Academic Press, New York, 1976, pp. 113-141.

2. S. KARLIN \& W. J. STUDDEN, Tchebycheff Systems: With Applications in Analysis and Statistics, Interscience, New York, 1966.

3. M. G. KREǏN \& M. A. RUTMAN, "Linear operators leaving invariant a cone in a Banach space," Uspehi Mat. Nauk, v. 3, 1948, pp. 3-95; English transl., Amer. Math. Soc. Transl. (1), v. 10, 1962, pp. 199-325.

4. J. T. SCHWARTZ, Nonlinear Functional Analysis, Gordon and Breach, New York, 1969. 